\title{
Study of Functionalized Gold Nanoparticles with Anti-gp63 IgG Antibody for the Detection of Glycoprotein gp63 in Membrane Surface of Leishmania Genus Parasites
}

\author{
Pedro Rodríguez ${ }^{1,2}$, Humberto Rojas ${ }^{1,3}$, Miguel Medina ${ }^{1,4}$, Jazzmin Arrivillaga ${ }^{4}$, Yosue Francisco ${ }^{1,2}$, \\ Fracehuli Dager $^{2,5}$, Vincent Piscitelli ${ }^{6,7}$, Manuel Caetano ${ }^{6,7}$, Alberto Fernández ${ }^{6,7}$, Jimmy Castillo ${ }^{6,7}$ \\ ${ }^{1}$ Centro de Microscopia Electrónica, Facultad de Ciencias, Universidad Central de Venezuela, Caracas, Venezuela \\ ${ }^{2}$ Escuela de Biología, Facultad de Ciencias, Universidad Central de Venezuela, Caracas, Venezuela \\ ${ }^{3}$ Escuela de Física, Facultad de Ciencias, Universidad Central de Venezuela, Caracas, Venezuela \\ ${ }^{4}$ Departamento de Biología, Universidad Simón Bolívar, Caracas, Venezuela \\ ${ }^{5}$ Instituto de Biología Experimental, Facultad de Ciencias, Universidad Central de Venezuela, Caracas, Venezuela \\ ${ }^{6}$ Laboratorio de Espectroscopia Laser, Facultad de Ciencias, Universidad Central de Venezuela, Caracas, Venezuela \\ ${ }^{7}$ Escuela de Química, Facultad de Ciencias, Universidad Central de Venezuela, Caracas, Venezuela \\ Email: pedroj.rodriguez@ciens.ucv.ve
}

Received May 15, 2013; revised June 16, 2013; accepted July 3, 2013

Copyright (C) 2013 Pedro Rodríguez et al. This is an open access article distributed under the Creative Commons Attribution License, which permits unrestricted use, distribution, and reproduction in any medium, provided the original work is properly cited.

\begin{abstract}
In this work we present the preparation and functionalization of gold nanoparticles (AuNPs) for the detection of presence of gp63 glycoprotein in the surface of Leishmania genus parasites. AuNPs were prepared by induced laser ablation in a clean and biologically suitable media. The nanoparticles were functionalized with anti-gp63 $\lg$ antibody in order to study the interaction with the glycoprotein component gp63 $(63 \mathrm{kDa})$ present on the membrane surface of Leishmania genus parasites. The functionalized AuNPs showed potential as a spectrometric indicator of the parasite existence, both by the detection of the presence of the gp63 in solution and through the specific interaction with the parasites in vitro. The specificity of the study opens a new line of research on the use of modified nanoparticles in the development of a fast and easy assay for Leishmaniosis diagnostics.
\end{abstract}

Keywords: Leishmania; Nanoparticles; Surface Plasmon Resonance; gp63 Protein

\section{Introduction}

Nanotechnology appears to be a useful and promissory tool in biodiagnostics. The use of functionalized nanoparticles is widely applied to detect presence of biomolecules [1,2], cells and metallic ions [3-8]. Also, they have been used recently to study interactions between molecules. These devices are frequently faster, simpler and cheaper than conventional detection methods. Most of the devices developed are based in the study of Surface Plasmon Resonance (SPR) response of the Functionalized Nanoparticles (FNps) to the changes in the local environment. This response is detected as variations in the SPR extinction band using spectrometer or in the most sensitive cases by variations in the color of the sample. The interaction of nanoparticles with organic molecules is unspecific; the specificity of the sensor is achieved by the recovering of the nanoparticle with anti- body molecules that can interact with a very specific target. The changes observed in the extinction band of the nanoparticle recovered with the receptor molecule are due to variations in the environment surrounding the nanoparticles or the association and posterior aggregation induced by target molecules present in the media. In recent years, metallic nanoparticles are been used as vehicle to detect specific biological targets. It has been used SPR to detect the hybridization of cDNA-coated gold nanoparticles with a surface-bound RNA [9]. These cDNA-coated gold nanoparticles are used to detect ssDNA down to a concentration of $1 \mathrm{fM}$ in a volume of 25 zeptomoles. Also it has been developed a method to amplify the wavelength shift observed from localized surface plasmon resonance (LSPR) bioassays in gold nanoparticle-labeled antibodies [10]. This work involves detecting surface-bound analytes using gold nanoparticle conjugated antibodies as a way to enhance SPR shifts for 
more sensitive detection of low-concentration analytes. Gold nanoparticles coupled with receptor molecule $(\beta$ amiloyd) have been used as a colorimetric sensor able to demonstrate a relationship within misfolding and aggregation of proteins involved in Alzheimer's disease [2]. Gold nanoparticles coupled to lectin concanavalin A and agglutinin have also been used in order to study the specificity of this device to three cell lines expressing glycosyl complexes at their surfaces [3]. Parasite infections are spread all over the planet, especially in tropical regions, making the population under poverty or with poor conditions of life their most vulnerable target. Leishmaniosis is a vector-borne infectious disease that affects 88 countries with an estimated incidence of two million new cases each year. Leishmaniosis is caused by a protozoan parasite of the Leishmania genus which has the sandfly - or phlebotomous of the genus Lutzomyia as its vector in the new world and Phlebotomous in the old world. About 20 Leishmania species are responsible for a wide range of clinical manifestations, some of them are fatal if left untreated [11]. With an expanding endemicity, an estimated 350 million people at risk and $2,357,000$ disability-adjusted life years lost, leishmaniosis is becoming a worldwide re-emerging public health problem. In spite of this, its study has been neglected. One intriguing aspect of leishmaniosis is the wide spectrum of distinct clinical manifestations that include visceral, cutaneous, mucocutaneous, and diffuse cutaneous leishmaniosis [12]. Over 70 species of Lutzomyia are suspected to be implicated in the transmission of eight Leishmania species known to infect dogs in South America [13]. Lutzomyia longipalpis is the most common vector for visceral leishmaniosis, and as many as 100 species of the same genus are responsible for cutaneous and muco-cutaneous leishmaniosis. In Venezuela, leishmaniosis control begins by having relevant epidemicological information leading to the design of a social assistance program to treat infected people. Who are they? What's their age and living conditions? And other relevant information is crucial for the success of this program. Citizens between 0 and 29 years old living in small cities or in the countryside are the most vulnerable segment of the population. In 1995 the Pan-American Health Organization reported the existence of visceral, cutaneous, and muco-cutaneous leishmaniosis in Venezuela [14]. The most affected area in this country corresponds to the Andes region, where Táchira, Mérida and Trujillo states concentrate more than $60 \%$ of the infected population, with small farmers and villages population being the most vulnerable targets. Until today, the most common methods to detect Leishmaniosis infection include microscopic view, indirect immunofluorescence (using an anti-Leishmania $\operatorname{IgG}$ ) confirmed by western blotting, positive response to patients after antimonial treatment ( $\mathrm{N}$-methylglutamine) or liposomic
B amphotericine [15], indirect immunoperoxydase method [16], polymerase chain reaction [17], serology (ELISA) and lymphoproliferative response [18]. In this way, a sensible, cheap and reliable method for the diagnosis of Leishmaniosis occurrence is of great interest in order to improve the knowledge of the illness population prevalence and help local health policy decisions. The aim of the present work is to prepare functionalized gold nanoparticles (FAuNPs) which could be able to detect a membrane surface antigen, the $63 \mathrm{kDa}$ glycoprotein named as gp63 with protease activity $[19,20]$, which is expressed on the surface of several Leishmania species, includs twelve different strains of Leishmania, including L. major, L. donovani, L. infantum, L. tropica, L. mexicana, L. amazonensis, L. braziliensis, and L. enriettii [21]. In L. major LEM 513, the cell is covered by approximately $5 \times 105$ copies of gp63 which correspond to about $1 \%$ of the total proteins of the cell [22] and the protein is anchored in the membrane by a glycophospholipid which is structurally homologous to the anchor of the variante surface glycoprotein (VSG) of Trypanosoma brucei [19]. Two reports that analyse the peptide maps of iodinated gp63 of $L$. donovani, $L$. major, and $L$. tropica, and L. donovani, L. major and L. braziliensis [21] demonstrate that the primary structures of the proteins are highly conserved. The interaction of the functionalized nanoparticles was tested in vitro. The results obtained are the first steps towards the development of a methodology able to provide very important information about Leishmaniosis occurrence in the third world countries, focusing our efforts in Venezuela.

\section{Materials and Methods}

\subsection{Gold Nanoparticle and Antibody Binding}

Mouse IgG anti-Leishmania major surface protease gp63 (from now on named anti-gp63) was obtained from AbD serotec OBT2004. Polyethylene glycol (PEG) from Sigma-Aldrich. Solutions were prepared using deionized water $18 \mathrm{~m} \Omega$ filtered through $0.45 \mu \mathrm{m}$ Millipore filter. Gold nanoparticles were prepared by Laser Ablation of a thin film of gold immersed in an aqueous solution in PEG, using the first harmonic of a Nd:YAG laser (1064 $\mathrm{nm}$ ) at a frequency rate of $10 \mathrm{~Hz}$ with a pulse duration of $8 \mathrm{~ns}$ and $145 \mathrm{~mJ} /$ pulse of power. The irradiation of the metal results in fast removal of the material that is confined to laser spot. The ejected nanoparticles remain in the liquid forming the colloidal solution that exhibited long term stability. The size-control was obtained by proper tuning of the surfactant concentration, the laser fluence and numbers of shot [23]. The size and morphology of the AuNPs were evaluated using a transmission electron microscopy (TEM) JEOL model JEM-1220 operated at $120 \mathrm{kV}$. The samples for TEM observation 
were prepared by placing a drop of nanoparticle solution on colodion coated copper grids and evaporating it in air at room temperature. Images were recorded by using Kodak 4489 film. The extinction spectra of the plasmonic resonance band of the AuNPs were detected using an Ocean Optics S2000 spectrometer. The AuNPs obtained as described above are gold nanoparticles surrounded by PEG. The PEG acts like surfactant maintaining the AuNPs dispersed in the solution to avoid their aggregation; additionally the PEG permits to obtain a correct ulterior orientation of IgG. In fact, IgG will bind to the PEG by interaction at the C-terminus of the heavy chains leaving the fragment of the IgG antigen binding (FAB) towards the surrounding solution. To obtain the FAuNPs the PEG covered AuNPs were left in contact with a 1:100 anti-gp63 solution $(1 \mathrm{mg} / \mathrm{ml})$ for $24 \mathrm{~h}$.

\subsection{Parasite Culture}

Leishmania major promastigotes were cultured at $27^{\circ} \mathrm{C}$ by serial passages in Schneider's insect medium $\mathrm{pH} 7.4$ supplemented with $10 \%$ inactivated fetal bovine serum (FBS). Cells were harvested in late log phase $\left(4-5 \times 10^{6}\right.$ parasites $\mathrm{ml}^{-1}$ ) by centrifugation at $2500 \times \mathrm{g}$ at $4^{\circ} \mathrm{C}$ for 10 minutes, and washed twice with phosphate-buffered saline (PBS, $10 \mathrm{mM}$ phosphate; $\mathrm{pH} 7.4 ; 2.7 \mathrm{mM} \mathrm{KCl}$; $137 \mathrm{mM} \mathrm{NaCl})$.

\subsection{Parasite Immobilization}

L. major promastigotes were immobilized in a $50 \mathrm{ml}$ sterile culture FALCON ${ }^{\circledR}$ flask. This was carried out by the following procedure: an L-polylisine solution (1 $\mathrm{mg} / \mathrm{ml}$ ) in PBS was added to the flask, enough to cover the entire flat surface, and after 30 minutes incubation, the solution discarded and the flask rinsed twice with PBS pH 7.4. A suspension of washed parasites in PBS was added to the culture flask and left for 15 minutes, or until all the parasites were adhered to the flask bottom. The cells were fixed with 3\% para-formaldehyde solution in PBS for 10 minutes, washed in PBS, rinsed for 5 minutes with ammonium chloride $50 \mathrm{mM}$ in PBS and finally rinsed twice with PBS.

\section{4. gp63 Protein Purification}

The procedure for gp63 purification was carried out as described [18] with slight modifications [21]. Briefly, $1 \times$ $10^{9}$ washed promastigotes were mixed and extracted at $0^{\circ} \mathrm{C}$ for 10 minutes in $10 \mathrm{ml}$ of TBS containing $2 \%(\mathrm{w} / \mathrm{v})$ of precondensed Triton X-114 (Sigma), and sedimented at $50.000 \mathrm{xg}$ for 30 minutes at $4^{\circ} \mathrm{C}$. The supernatant was incubated for 5 minutes at $30^{\circ} \mathrm{C}$ to induce the aggregation of the Triton X-114 micelles and the solution centrifuged at $5000 \mathrm{xg}$ for 10 minutes at $28^{\circ} \mathrm{C}$ to separate the phases.
The detergent phase was diluted 1:5 with Triton X100

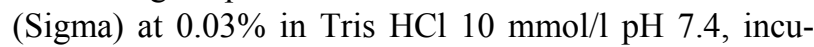
bated for 10 minutes at $4{ }^{\circ} \mathrm{C}$ and centrifuged at $5000 \mathrm{xg}$ for 15 minutes at the same temperature until clarification. The detergent-enriched phase containing integral membrane protein was finally diluted to 1:20 with Triton X-

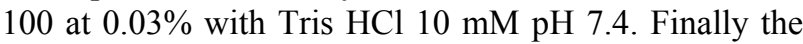
extracted surface antigen was purified through a column of anionic exchange DEAE52 and the product analyzed by SDS PAGE to verify the purity of the sample. The purify antigen was kept in small aliquots at $-20^{\circ} \mathrm{C}$ until used.

\subsection{Light Interferometer Microscopy}

Two kinds of samples were observed under the white light interferometer microscope, New View 600 model Zygo MetroPro. In one hand the control sample composed by Leishmania parasite cultives fixed onto glass plate which were left in contact with non-functionalized gold nanoparticles by 4 hours. In the other hand sample was composed by Leishmania parasite cultive fixed onto glass plates and left in contact with functionalized nanoparticles by 4 hours.

\section{Results}

Figure 1 shows the extinction spectra of the gold nanoparticles dispersed in water with PEG as surfactant. Typical extinction band with a maximum at $524 \mathrm{~nm}$ is observed.

This band corresponds with the SPR of the AuNPs of approximately $30 \mathrm{~nm}$ in size. The inset in the figure shows the electron micrograph of the obtained particles. The size of the nanoparticles was obtained by averaging the size of the particles observed in twenty electron microscope images, assuming all to be spherical in shape.

\subsection{Nanoparticles Functionalization}

Figure 2 shows the corrected absorption spectra for solutions of non-functionalized gold nanoparticles (AuNPs) with PEG and the FAuNPs. This plot shows a clear red shift of approximately $4 \mathrm{~nm}$ in the SPR band for the FAuNPs compared with the AuNPs with PEG spectra. This red shift in the band is a typical consequence of the variation in the local environment of the nanoparticle due to the covering of its surface with anti-gp63 molecules.

\subsection{Interaction of Functionalized Nanoparticles with gp63}

The interaction of the AuNPs-PEG-anti-gp63 array (FAuNPs) was studied by putting in contact this array and the gp63 extract previously obtained. Solutions with 


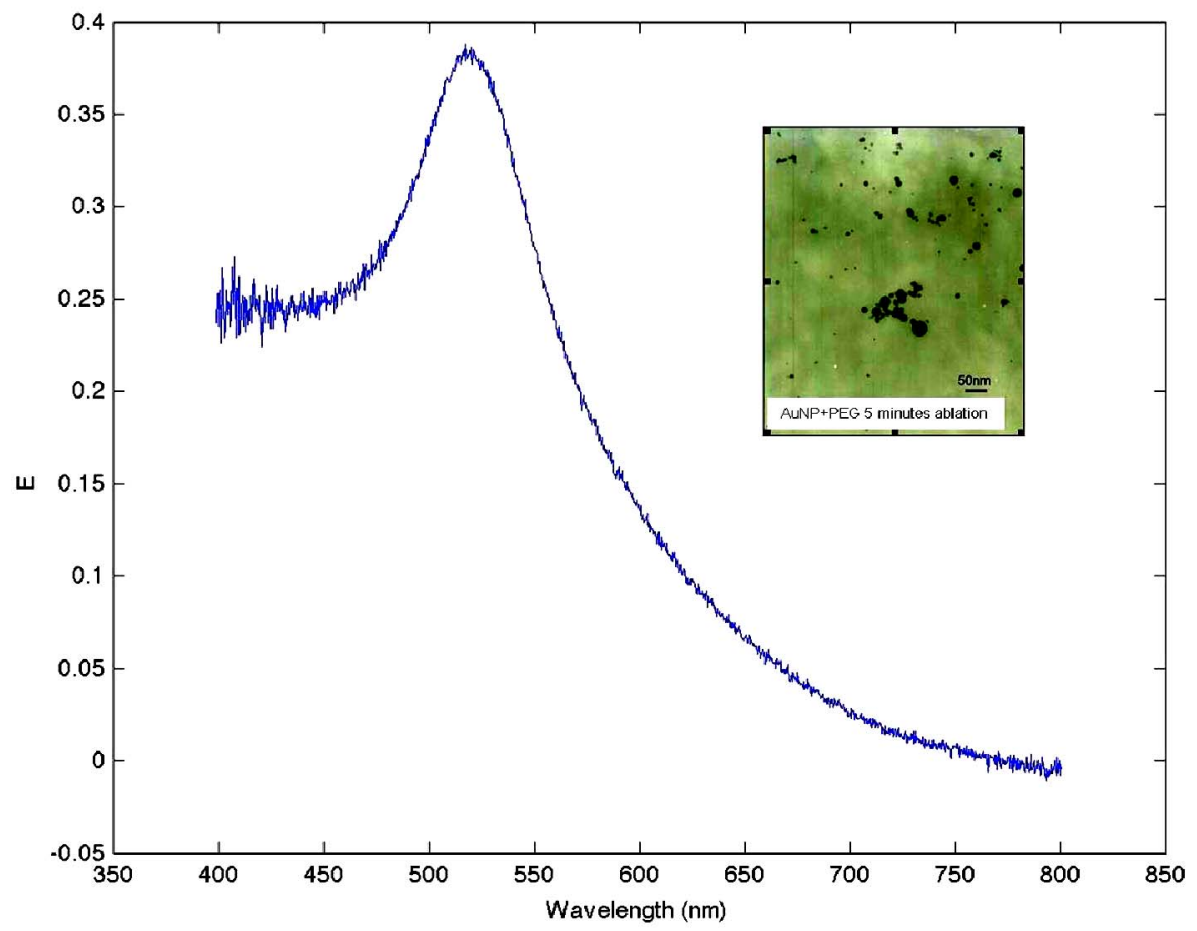

Figure 1. Extinction spectra of the gold nanoparticles dispersed in water with PEG as surfactant. In the inset electron micrograph of AuNPs.

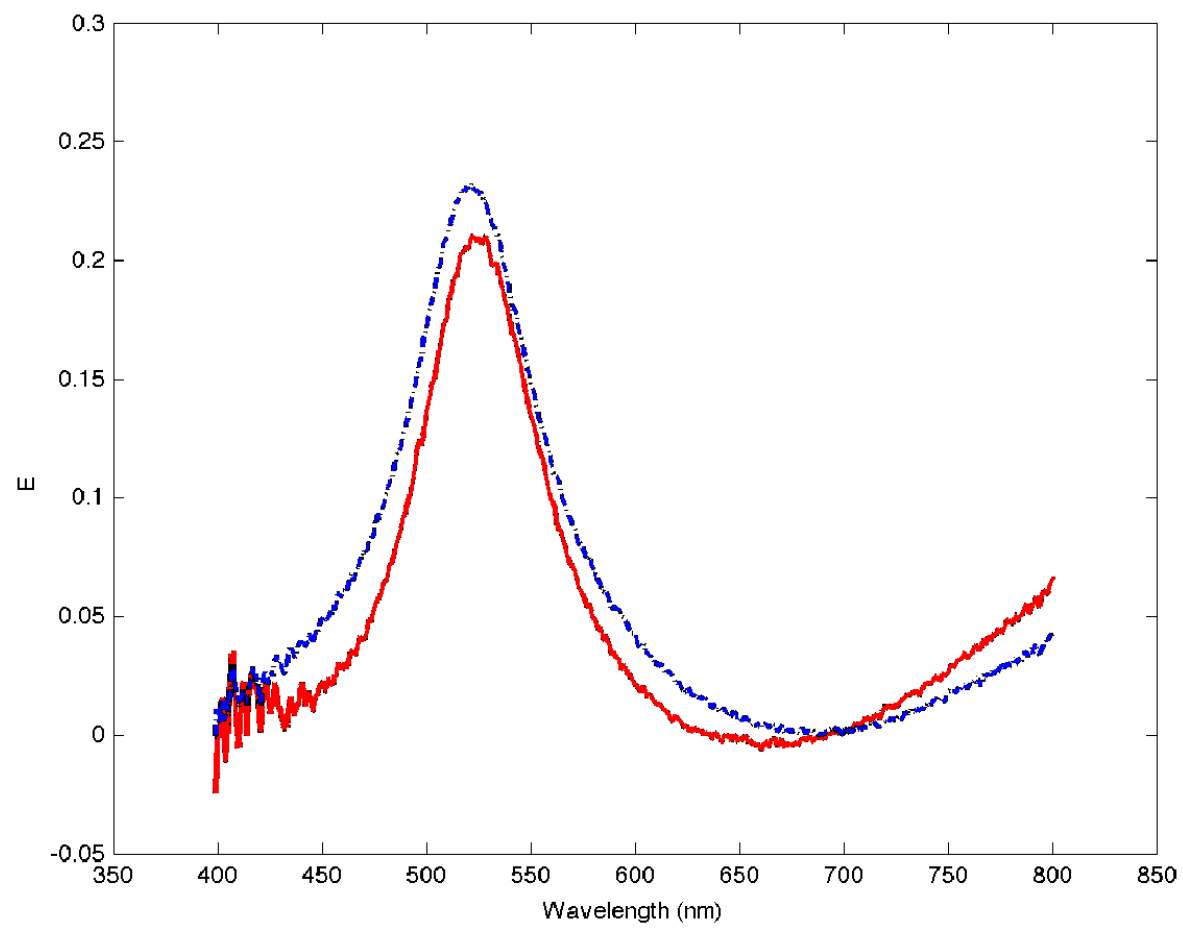

Figure 2. Corrected absorption spectra for solutions of AuNPs (blue and dashed line) with PEG and the FAuNPs (red and solid line).

$5 \mathrm{ml}$ of FAuNPs were mixed with additive volumes of the gp63 extract; starting from $100 \mu \mathrm{L}$ and increasing up to $900 \mu \mathrm{L}$. A negative control represented by non-func- tionalized AuNPs-PEG was left interact with the same gp63 antigen solution in order to discard any non specific reaction between PEG layer and the gp63 antigen. For 
this negative control no variation in the extinction spectra was observed. Figure 3 shows the corrected SPR spectra for the FAuNPs in contact with increasing amounts of gp63.

In Figure 4 the variation of the maximum SPR intensity is plotted as a function of the quantity of gp63 added. Both plots show a decrease in the SPR signal with higher quantities of gp63 present in the media. This reduction is due to the diminishing in number of the nanoparticles responsible of this band in the media. The interaction of the FAuNPs with the gp63 conduces to the aggregation of nanoparticles forming a new colloid of higher size and as a consequence, to the existence of fewer particles in the media that contribute to decrease in the SPR band intensity.

\subsection{Interaction of Functionalized Nanoparticles with Parasites}

AuNPs-PEG-anti-gp63 array was also tested in vitro.

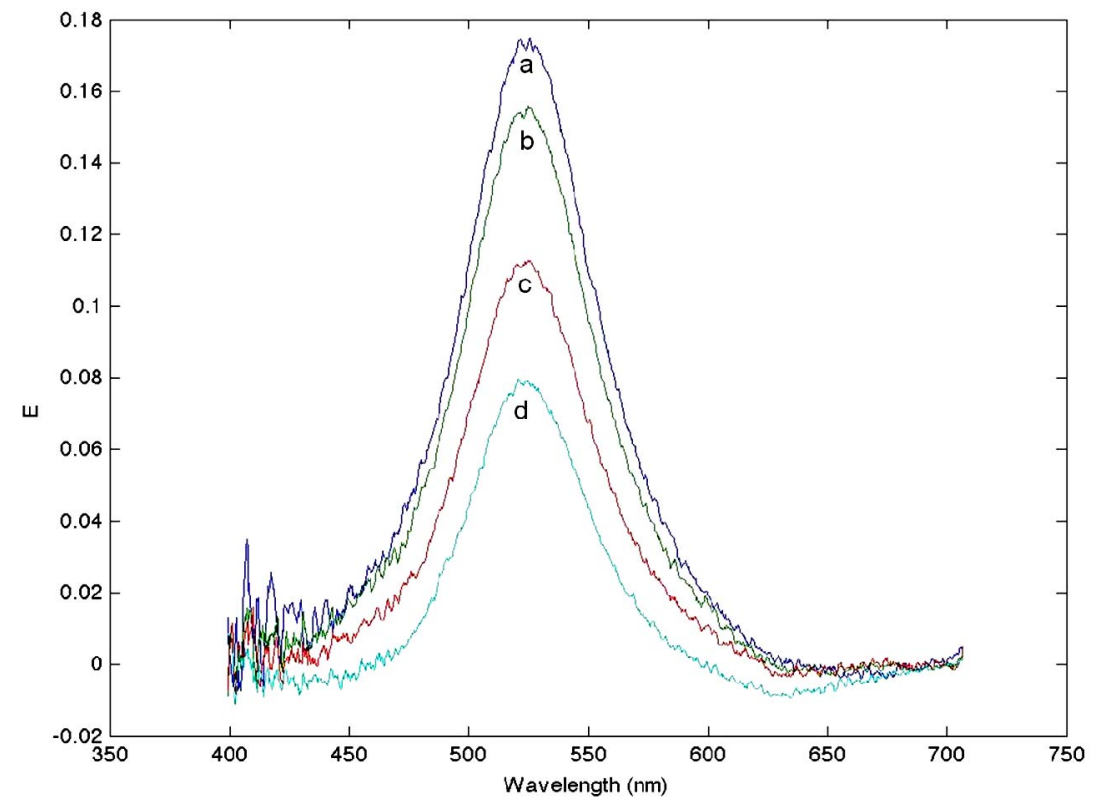

Figure 3. Corrected SPR spectra for the FAuNPs in contact with increasing amounts of gp63. Curve a: $100 \mu \mathrm{L}$, curve b: 200 $\mu \mathrm{L}$, curve c: $600 \mu \mathrm{L}$ and curve d: $900 \mu \mathrm{L}$.

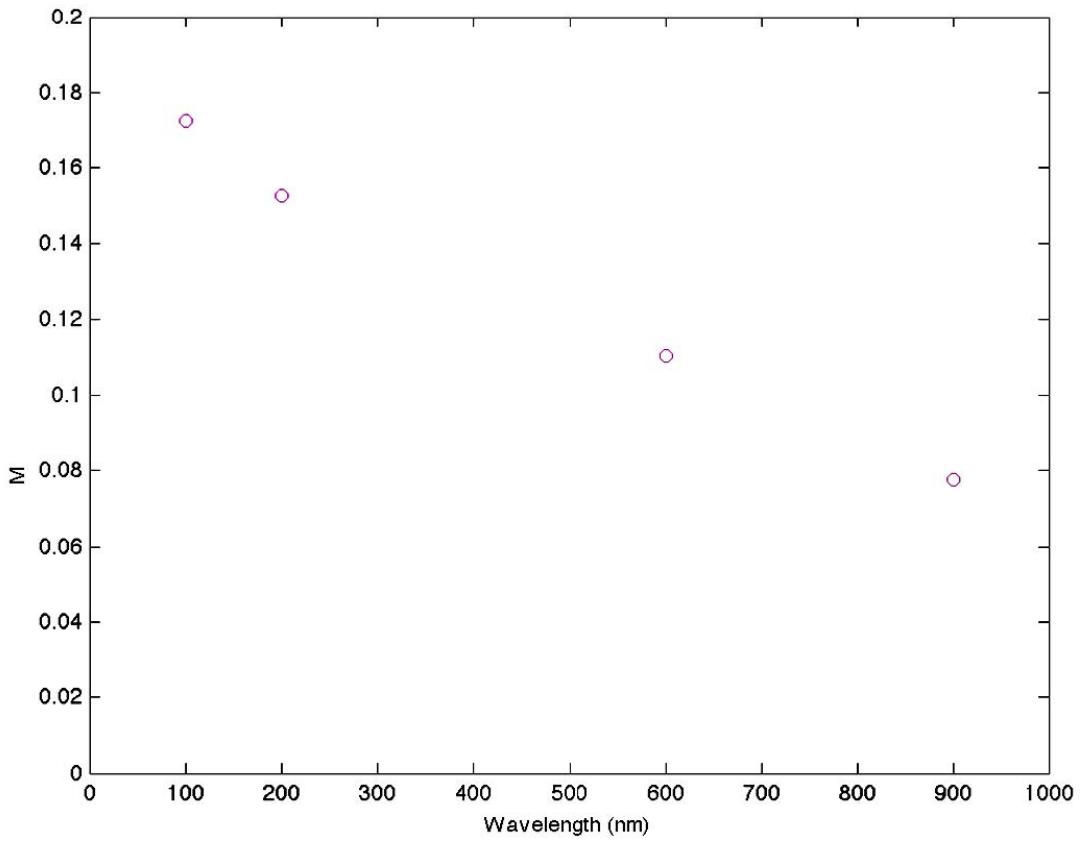

Figure 4. Variation of the maximum SPR intensity is plotted as a function of the quantity of gp63 added. 
Immobilized promastigotes from $L$. major as described before were put in contact with FAuNPs and the extincttion spectra were recorded every hour during $48 \mathrm{~h}$ at $4^{\circ} \mathrm{C}$. A conspicuous loss of the suspension color was observed. In order to determine if the observed interaction leading to observed loss of color was specific and a consequence of the antigen/antibody (gp63/anti-gp63) interaction, a blank test represented by the same arrangement without parasite cells, was carried out obtaining no changes in the SPR spectra. Results are in Figure 5.

These results were confirmed using L. mexicana in similar conditions as before, with similar results to those obtained with L. major. Figure 6 shows images of parasites supported on glass plates after exposure to nonfunctionalized AuNPs (AuNPs, top) and functionalized AuNPs (FAuNPs, bottom).

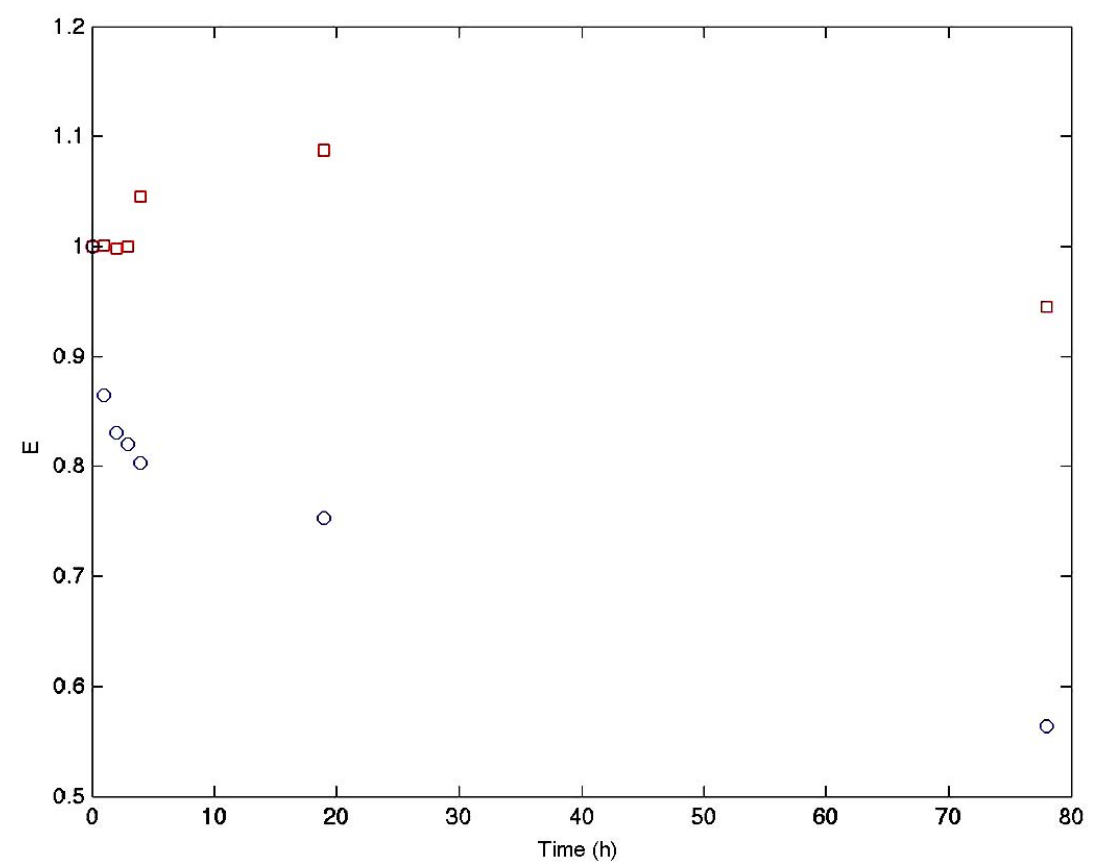

Figure 5. Variations in the SPR maxima for the FAuNPs in contact with immobilized L. major promastigotes cells (circles) and FAuNPs in contact with the Falcon ${ }^{\circledR}$ bottle without cells (squares).
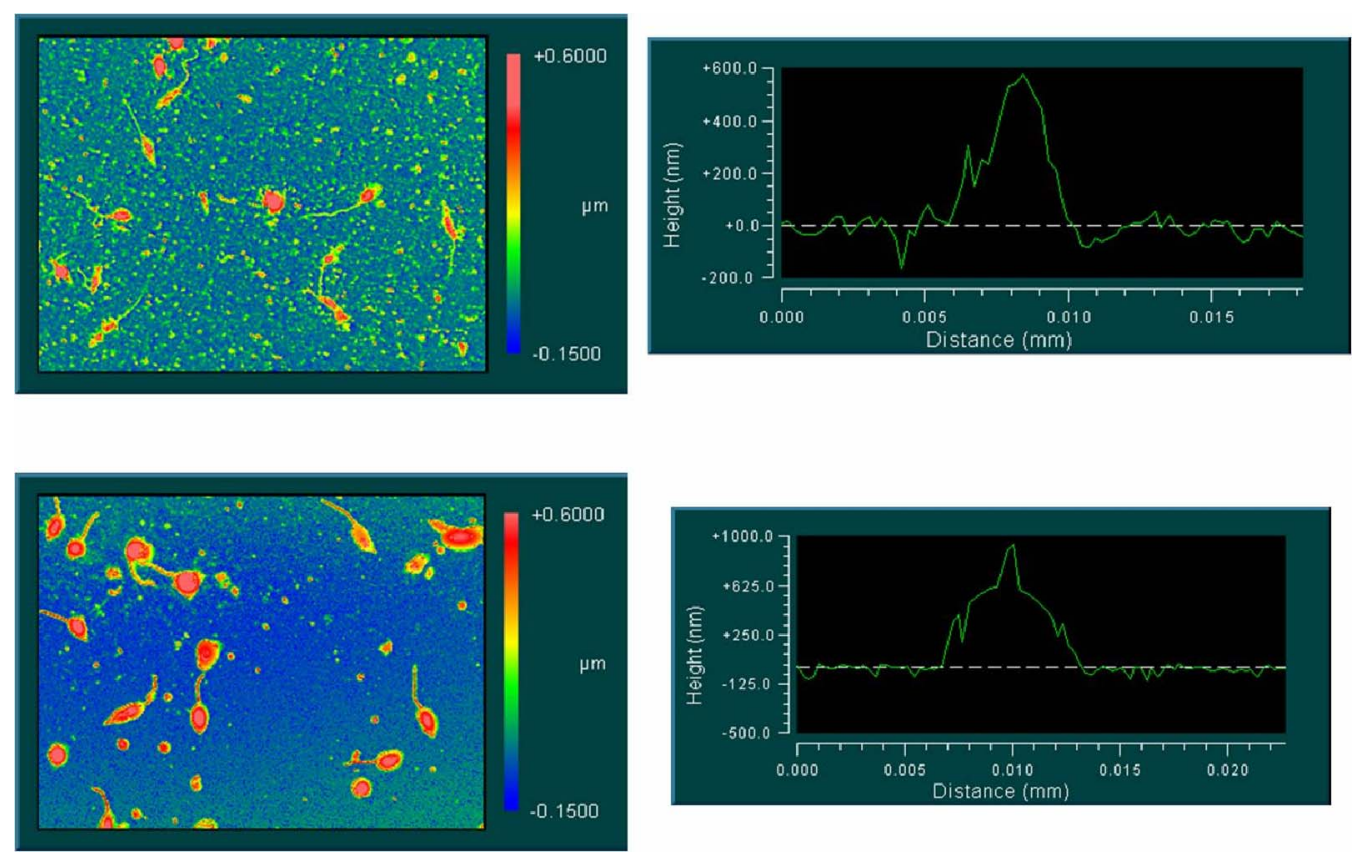

Figure 6. Interferometric images from parasites in contact with AuNPs (top) and FAuNPs (bottom). 
Parasites exposed to FAuNPs are coated by metallic nanoparticles and present a greater contrast than parasites exposed to non-functionalized AuNPs. The interferometric images draw a difference in the width and height of the parasites in these two experimental conditions. The observed difference could be due to the presence of nanoparticles adsorbed on the surface of the parasite.

\section{Discussion}

Interaction of functionalized gold nanoparticles for the detection of Leishmania genus parasites was studied. The functionalized nanoparticles proved to be highly sensible and selective in the detection of the gp63 surface membrane antigen both in solution and when exposed to immobilized Leishmania major and L. mexicana promastigotes. The effect shown that can be useful as a colorimetric indicator of the parasite existence. Corrected SPR spectra for the FAuNPs in contact with increasing amounts of gp63 showed a decrease in the SPR signal with higher quantities of gp63 present in the media. The synthesis by laser ablation is a simple and easy way to use technique for obtaining metallic nanoparticles. The ablation time and the energy applied to the metallic plate are two of the parameters that must be considered when performing the synthesis. In our experiments the union between the FAuNPs to promastigote both immobilized and in suspension, as well as to protein gp63, was evidenced. These tests were designed taking advantage of the spectroscopic changes that took place due to the aggregation effect of the metallic nanoparticles [22,24,25]. The registered changes were used to expose the interaction of the FAuNPs complex with the gp63 protein. In all our experiments we observed changes in the absorption spectra as a consequence of the FAuNPs-protein interaction. In all the tests a decrease in the absorption spectrum of the AuNP occurred, in particular in the experiments using a suspension of L. major and a pure extract of gp63, where a displacement of the absorption peak in the normalized spectra above a wavelength of $520 \mathrm{~nm}$ was observed. The spectroscopic changes due to the aggregation of the metallic nanoparticles have been reported previously in the literature. The development of biosignal detection methods with the use of plasmonic nanosensors has been mentioned [26]. The authors worked with the extinction spectrum of both gold and silver nanoparticles, defining the extinction as the capacity to obscure or extinguish certain wavelength. In this sense a shift of the maxima within the extinction spectra in experiments using FAuNPs has been previously observed [22]. The arrays of immobilized proteins over metallic nanoparticles have a high potentiality for the detection of specific cells. The report of the development of applied nanotechnology for the detection of microorganisms has been mentioned

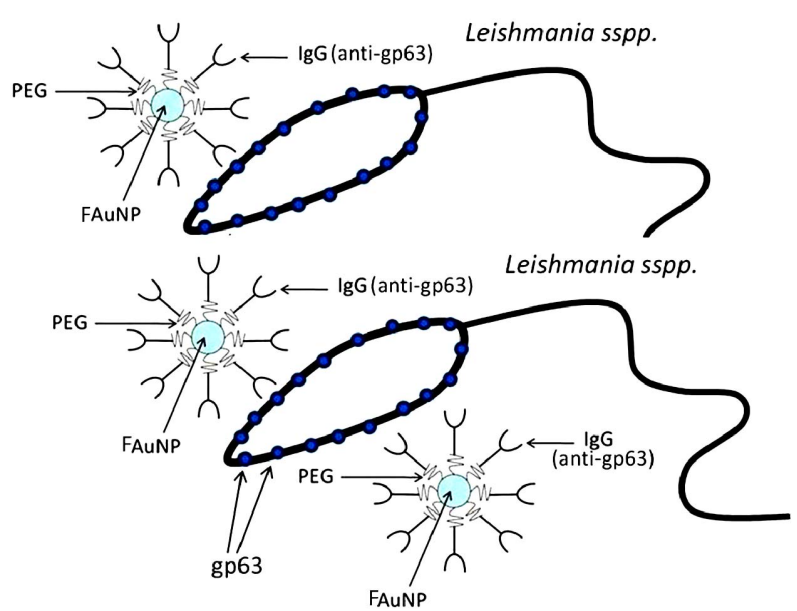

Figure 7. Model proposed for interaction between FAuNPs and Leishmania sp. promatigotes (PEG: Polyethylene glycol. IgG (anti-gp63): Immunoglobulin $G$ against gp63 Leishmania genus protein. gp63: Glycoprotein $63 \mathrm{kDa}$ from Leishmania genus. FAuNP: Functionalized gold nanoparticle). PS: Both promastigotes and FAuNP are not proportionally related in size.

[27], one of the methods authors mention is the spectral chance due to metallic nanoparticles aggregation and that method can be used for direct detection of suspended cells, viruses or microorganism related molecules. The aggregation of the metallic nanoparticles generates a change in their geometric form. When a different tridimensional form is obtained, a change in the plasmonic resonance occurs causing a colorimetric change $[24,28$, 29]. This phenomenon is of interest for the present research project since our objective is to take advantage of the changes in spectral properties for the development of a biotest for the early detection of tropical parasite diseases. In the present study we have verified the capacity of the FAuNPs to couple to parasites both immobilized and in suspension. Also we have observed its ability to join two antigens in solution, as in the case of the gp63 protein present on the surface of Leishmania major. Taking this into account for the design of a field biotest, we would observe a discoloration of nanoparticles suspension in the presence of the parasites from samples. Taken together, our results allow us to propose the following interaction scheme between the functionalized gold nanoparticles and Leishmania sp. promastigotes (see Figure 7).

\section{Acknowledgements}

This work was supported by a grant from Venezuelan National Found for Science and Technology. FONACIT Project Number 2007000960. Pedro Rodriguez dedicates this work to Drs. Mitsuo Ogura (1928-2000) and Tatiana Mérida (1941-2006) In memoriam. 


\section{REFERENCES}

[1] A. Gultekin, A. Ersoz, D. Hur, N. Sarızlu, A. Denizli and R. Say, "Gold Nanoparticles Having Dipicolinic Acid Imprinted Nanoshell for Bacillus Cereus Spores Recognition," Applied Surface Science, Vol. 256, No. 1, 2009, pp. 142-148. doi:10.1016/j.apsusc.2009.07.097

[2] C. Wang, J. Wang, D. Liu and Z. Wang, "Gold Nanoparticle-Based Colorimetric Sensor for Studying the Interactions of $\beta$-Amyloid Peptide with Metallic Ions," Talanta, Vol. 80, No. 5, 2010, pp. 1626-1631. doi:10.1016/j.talanta.2009.09.052

[3] J. Wang, T. Duan, L. Sun, D. Liu and Z. Wang, "Functional Gold Nanoparticles for Studying the Interaction of Lectin with Glycosyl Complex on Living Cellular Surfaces," Analytical Biochemistry, Vol. 392, No. 1, 2009, pp. 77-82. doi:10.1016/j.ab.2009.05.036

[4] J. Kneipp, H. Kneipp, B. Wittig and K. Kneipp, "Novel Optical Nanosensors for Probing and Imaging Live Cells," Nanomedicine, Vol. 6, No. 2, 2010, pp. 214-226. doi:10.1016/i.nano.2009.07.009

[5] M. Velasco-Garcia, "Optical Biosensors for Probing at the Cellular Level: A Review of Recent Progress and Future Prospects," Seminars in Cell \& Developmental Biology, Vol. 20, No. 1, 2009, pp. 27-33. doi:10.1016/j.semcdb.2009.01.013

[6] T. Vo-Dinh, "Nanosensing at the Single Cell Level," Spectrochimica Acta Part B: Atomic Spectroscopy, Vol. 63, No. 2, 2008, pp. 95-103. doi:10.1016/j.sab.2007.11.027

[7] H. Patra, S. Banerjee, U. Chaudhuri, P. Lahiri and A. Dasgupta, "Cell Selective Response to Gold Nanoparticles," Nanomedicine, Vol. 3, No. 2, 2007, pp. 111-119. doi:10.1016/j.nano.2007.03.005

[8] B. Leng, L. Zou, J. Jiang and H. Tian, "Colorimetric Detection of Mercuric Ion $\left(\mathrm{Hg}^{2+}\right)$ in Aqueous Media Using Chemodosimeter-Functionalized Gold Nanoparticles," Sensors and Actuators B: Chemical, Vol. 140, No. 1, 2009, pp. 162-169. doi:10.1016/j.snb.2009.03.074

[9] I. Sendroiu, L. Gifford, A. Lupták and M. Corn, "Ultrasensitive DNA Microarray Biosensing via in Situ RNA Transcription-Based Amplification and Nanoparticle-Enhanced SPR Imaging," Journal of the American Chemical Society, Vol. 133, No. 12, 2011, pp. 4271-4273. doi:10.1021/ja2005576

[10] W. Hall, N. Ngatia and R. Van Duyne, "LSPR Biosensor Signal Enhancement Using Nanoparticle-Antibody Conjugates," The Journal of Physical Chemistry C, Vol. 115, No. 5, 2011, pp. 1410-1414. doi:10.1021/jp106912p

[11] P. Borst and M. Oullette, "New Mechanisms of Drug Resistance in Parasitic Protozoa," Annual Review of Microbiology, Vol. 49, 1995, pp. 427-460. doi:10.1146/annurev.mi.49.100195.002235

[12] F. Oliveira, R. Jochim, J. Valenzuela and S. Kamhawi, "Sand Flies, Leishmania, and Transcriptome-Borne Solutions," Parasitology International, Vol. 58, No. 1, 2009, pp. 1-5. doi:10.1016/j.parint.2008.07.004

[13] F. Dantas-Torres, "Canine Leishmaniosis in South America," Parasit Vectors, Vol. 2, No. S1, 2009. doi:10.1186/1756-3305-2-S1-S1
[14] Pan American Health Organization, Cuaderno Técnico No. 44, Washington DC, 1996.

http://www1.paho.org/Spanish/AD/DPC/CD/epi-y-contro 1.pdf

[15] F. Figueiredo, M. Madeira, R. Menezes, R. Pacheco, M. Pires, M. Furtado, A. Pinto and T. Schubach, "Efficacy of an Indirect Immunofluorescence Test in the Diagnosis of Canine Leishmaniosis," The Veterinary Journal, Vol. 186, No. 1, 2010, pp. 123-124. doi:10.1016/j.tvj1.2009.06.030

[16] R. Etges, J. Bouvier, R. Hoffman and C. Bordier, "Evidence That the Major Surface Proteins of Three Leishmania Species Are Structurally Related," Molecular and Biochemical Parasitology, Vol. 14, No. 2, 1985, pp. 141-149. doi:10.1016/0166-6851(85)90033-7

[17] V. Colomer-Gould, L. Galvao Quintao, J. Keithly and N. Nogueira, "A Common Major Surface Antigen on Amastigotes and Promastigotes of Leishmania Species," The Journal of Experimental Medicine, Vol. 162, No. 3, 1985, pp. 902-916. doi:10.1084/jem.162.3.902

[18] J. Bouvier, R. Etges and C. Bordier, "Identification and Purification of Membrane and Soluble Forms of the Major Surface Protein of Leishmania Promastigotes," The Journal of Biological Chemistry, Vol. 260, No. 29, 1985, pp. 15504-15509.

[19] C. Bordier, R. Etges, J. Ward, M. Turner and M. Cardoso de Almeida, "Leishmania and Trypanosoma Surface Glycoproteins Have a Common Glycophospholipid Membrane Anchor," Proceedings of the National Academy of Sciences of the USA, Vol. 83, No. 16, 1986, pp. 5988-5991. doi:10.1073/pnas.83.16.5988

[20] R. Etges, J. Bouvier and C. Bordier, "The Major Surface Protein of Leishmania Promastigotes Is a Protease," The Journal of Biological Chemistry, Vol. 261, No. 20, 1986, pp. 9098-9101.

[21] A. Hernández, A. Rascón, S. Kutner, H. Roman and Z. Campos, "Relationships between Cell Surface Protease and Acid Phosphatase Activities of Leishmania Promastigote," Molecular Biology Reports, Vol. 18, No. 3, 1993, pp. 189-195. doi:10.1007/BF01674430

[22] C. Yonzon, D. Stuart, X. Zhang, A. McFarland, C. Haynes and R. Van Duyne, "Towards Advanced Chemical and Biological Nanosensors-An Overview," Talanta, Vol. 67, No. 3, 2005, pp. 438-448. doi:10.1016/j.talanta.2005.06.039

[23] M. Ortega, L. Rodriguez, J. Castillo, V. Piscitelli, A. Fernandez and L. Echevarria, "Thermo-Optical Properties of Gold Nanoparticles in Colloidal Systems," Journal of Optics, Vol. 10, No. 10, 2008, pp. 104024-104027.

[24] J. West, R. Drezek and N. Halas, "Nanotechnology Provides New Tools for Biomedical Optics," In: D. E. Reisner, Ed., Biotechnology Global Prospects, CRC Press, Taylor \& Francis Group, Boca Raton, 2009, pp. 261-268.

[25] N. Sanvicens and M. Marco, "Multifunctional Nanoparticles-Properties and Prospects for Their Use in Human Medicine," Trends in Biotechnology, Vol. 26, No. 8, 2008, pp. 425-433. doi:10.1016/j.tibtech.2008.04.005

[26] J. Anker, N. Jeffrey, W. Paige, O. Lyandres, N. Shah, J. Zhao and R. Van Duyne, "Biosensing with Plasmonic Nanosensors," Nature Materials, Vol. 7, 2008, pp. 442- 
453. doi:10.1038/nmat2162

[27] Ch. Kaittanis, S. Santra and J. M. Perez, "Emerging Nanotechnology-Based Strategies for the Identification of Microbial Pathogenesis," Advanced Drug Delivery Reviews, Vol. 62, No. 4-5, 2010, pp. 408-423. doi:10.1016/j.addr.2009.11.013

[28] P. Skottrup, M. Nicolaisen and A. Justesen, "Towards
On-Site Pathogen Detection Using Antibody-Based Sensors," Biosensors and Bioelectronics, Vol. 24, No. 3, 2008, pp. 339-348. doi:10.1016/j.bios.2008.06.045

[29] S. Nash, S. Jana, M. Pradhan and T. Pal, "Ligand-Stabilized Metal Nanoparticles in Organic Solvent," Journal of Colloid and Interface Science, Vol. 341, No. 2, 2010, pp. 333-352. doi:10.1016/j.jcis.2009.09.049 